\title{
Hypoxic Breast Cancer Cell-Derived Exosomal SNHGI Promotes Breast Cancer Growth and Angiogenesis via Regulating miR-2I6b-5p/JAK2 Axis
}

\author{
Gaosai Dai' \\ Yupeng Yang ${ }^{2}$ \\ Shuhao Liu $^{3}$ \\ Huantao Liu' \\ 'Department of Breast Surgery, Qilu \\ Hospital of Shandong University, Jinan, \\ Shandong, 2500I2, People's Republic of \\ China; ${ }^{2}$ Department of Thyroid and \\ Breast Surgery, Jinan Zhangqiu District \\ Hospital of TCM, Jinan, Shandong, \\ 250200, People's Republic of China; \\ ${ }^{3}$ Department of General Surgery, Qilu \\ Hospital of Shandong University, Jinan, \\ Shandong, 250012, People's Republic of \\ China
}

Background: Hypoxia is an important process that involved in the tumor microenvironment. In addition, hypoxic tumor cell-derived exosomes could promote tumor growth and angiogenesis. Thus, we aimed to investigate whether exosomes could regulate tumor development and progression under hypoxia in breast cancer.

Methods: The level of SNHG1 in hypoxic breast cancer cells and exosomes derived from hypoxic breast cancer cells was determined by real-time qPCR assay. Bioinformatics prediction and dual-luciferase reporter assays were used to determine the interaction between SNHG1, miR-216b-5p and JAK2.

Results: We found that comparing with exosomes derived from normoxia breast cancer cells, exosomes derived from hypoxic breast cancer cells could promote the proliferation, migration and angiogenesis of human umbilical vein endothelial cells (HUVECs). In addition, SNHG1 level was significantly upregulated in exosomes derived from hypoxic breast cancer cells. Moreover, exosome-mediated delivery of SNHG1 siRNA3 markedly reversed the effects of exosome-mediated delivery of SNHG1 on HUVECs. Mechanically, SNHG1 could increase the level of JAK2 by competitively binding to miR-216b-5p. Additionally, exosome-mediated delivery of SNHG1 was found to promote breast cancer growth in vivo. Conclusion: Collectively, our study revealed that exosomal SNHG1 from hypoxic breast cancer cells could promote tumor angiogenesis and growth via regulating miR-216b-5p/JAK2 axis, suggesting that SNHG1 may serve as a potential therapeutic target for breast cancer.

Keywords: breast cancer, exosomes, tumor microenvironment, hypoxia, long noncoding RNAs

\section{Introduction}

Breast cancer is the most common malignancy among women worldwide. ${ }^{1}$ It is estimated that there are approximately 1.3 million new cases of breast cancer worldwide and almost 500,000 die from it every year. ${ }^{2}$ Recently, radiotherapy, chemotherapy and neoadjuvant systemic treatments are the main treatments for breast cancer. $^{3-5}$ However, the prognosis for patients with breast cancer remains worse. ${ }^{6,7}$ Therefore, it is critical for improving the diagnosis and therapies for patients with breast cancer.

It has been shown that tumor progression is closely associated with the continued crosstalk between tumor cells and the surrounding tumor
Correspondence: Huantao Liu Department of Breast Surgery, Qilu Hospital of Shandong University, Wenhuaxi Road 107, Jinan, Shandong. 2500 I2, People's Republic of China Email liuhuantao3I@I63.com 
microenvironment (TME) they live in. ${ }^{8,9}$ Hypoxia is a key microenvironment feature in many solid tumors. $^{10}$ In addition, hypoxia is related to tumor growth, increased angiogenesis and poor prognosis. ${ }^{11}$ The major mechanism mediating cellular responses to hypoxia is that hypoxia-inducible factors (HIF1a and HIF2a); they can activate the transcription of target genes that exhibit vital roles in tumor growth, angiogenesis and metastasis. ${ }^{12}$ Exosomes, 40-150 $\mathrm{nm}$ in size, is able to mediate intercellular communication between cancer cells and recipient cells by transferring bioactive molecules including mRNAs, microRNAs (miRNAs), long non-coding RNAs (lncRNAs) and proteins. $^{13,14}$ Evidence has shown that hypoxia could increase the release of exosomes from tumor cells, thereby promoting tumor intercellular communication at long distances. ${ }^{15}$

Blood vessels that surround the tumor cells are important components in the tumor microenvironment. ${ }^{8,16}$ Mao et al found that exosomes derived from hypoxic esophageal squamous cell carcinoma (ESCC) cells significantly promoted the proliferation and tube formation of in human umbilical vein endothelial cells (HUVECs). ${ }^{17}$ Additionally, Xue et al found that exosomal IncRNA UCA1 derived from hypoxic bladder cancer cells could facilitate bladder tumor growth and development. ${ }^{18}$ Guo et al found that hypoxic tumor-derived exosomal lncRNA UCA1 promoted angiogenesis and tumor growth in pancreatic $^{\text {cancer. }}{ }^{19}$

LncRNA SNHG1 has been reported to act as a tumor-promoter in many cancers, such as colorectal cancer, breast cancer and prostate cancer. ${ }^{20-22}$ Previous study showed that SNHG1 promoted the progression of breast cancer through regulating miR-573/LMO4 axis. $^{23}$ In addition, Zuo et al illustrated that SNHG1 level was increased in breast cancer cells under

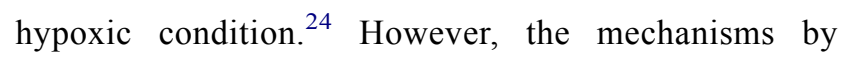
which exosomal lncRNA SNHG1 regulate tumor development and progression under hypoxia in breast cancer remain largely unclear.

In this study, we found that SNHG1 levels were significantly upregulated in exosomes derived from hypoxic breast cancer cells. In addition, exosomal SNHG1 from hypoxic breast cancer cells could promote tumor angiogenesis and growth. These findings may help to understand the diagnosis and treatment of breast cancer.

\section{Materials and Methods Cell Culture and Cell Transfection}

HUVECs, MDA-MB-231 and MCF-7 and 293T cells were obtained from American Type Culture Collection (ATCC) and cultured in DMEM plus $10 \%$ fetal bovine serum (FBS) in a humidified atmosphere of $5 \% \mathrm{CO}_{2}$ at $37^{\circ} \mathrm{C}$. For hypoxia stimulation, MDA-MB-231 and MCF-7 cells were cultured in a hypoxia chamber with $1 \% \mathrm{O}_{2}$ for $24 \mathrm{~h}$.

Three small interfering RNA (siRNA) oligonucleotides against SNHG1 (si-SNHG1-1, si-SNHG1-2, si-SNHG1-3), siRNA negative control (siRNA-ctrl), mimics control (mimics-ctrl), and miR-216b-5p mimics were obtained from Ribobio (Guangzhou, China). MDA-MB-231 and MCF-7 cells were transfected these plasmids using Lipofectamine 3000 (Invitrogen). si-SNHG1-1: 5'CAGTAAGTTCTTACGGCCATTTCTT-3'; si-SNHG1-2: 5'-CAACAAATGTCAGGGCCCTATTGAT-3'; si-SNHG1 -3: 5'-GCATATGGCTGAACTTTCAAGTGAT-3'; siRNActrl: 5'-GCCATGTTGTGACTTCGCCATCTAA-3'.

\section{Real-Time qPCR (RT-qPCR) Analysis}

Total RNA was extracted by the TRIpure Total RNA Extraction Reagent (ELK Biotechnology). Later on, the EntiLink $^{\mathrm{TM}}$ First Strand cDNA Synthesis Kit (ELK Biotechnology) was used for reverse transcription with 500 ng RNA. After that, RT-qPCR was performed by the QuantStudio 6 Flex System (Life Technologies) using the EnTurbo $^{\mathrm{TM}}$ SYBR Green PCR SuperMix (ELK Biotechnology). The primers were as follows: actin: 5'GTCCACCGCAAATGCTTCTA-3' (Forward); 5'TGCTGTCACCTTCACCGTTC-3' (Reverse). SNHG1: 5'-CTGGTGAAGGAATGGGACAAG-3' (Forward); 5'CCTTTGAGCCAAGCAGGTTAT-3' (Reverse).

\section{Exosome Isolation and Identification}

The ultracentrifugation method was used to isolate the exosomes from breast cancer cells. Briefly, the cell supernatant was collected and centrifuged at $300 \times \mathrm{g}$ for $10 \mathrm{~min}, 2000 \times$ $\mathrm{g}$ for $10 \mathrm{~min}, 10,000 \times \mathrm{g}$ for another $30 \mathrm{~min}$. After that, the supernatant was ultracentrifuged at $140,000 \times \mathrm{g}$ for $70 \mathrm{~min}$ twice. Later on, the supernatant was removed, and the pellet was obtained.

The size distribution of exosomes was determined by Nanoparticle Tracking Analysis (NTA) with a ZetaView nanoparticle tracking analyzer instrument (Particle Metrix).

The exosome pellet was fixed in $2.5 \%$ glutaraldehyde and then spotted onto a copper grid. After that, the grid 
was stained with $1 \%$ phosphotungstic acid for $3 \mathrm{~min}$. Subsequently, morphologies of the samples were observed by a HITACHI transmission electron microscope (TEM).

\section{Western Blot Assay}

Proteins were lysed using RIPA lysis buffer (ASPEN) and quantified by a BCA kit (Beyotime). After that, the equal amounts of proteins $(30 \mu \mathrm{g})$ were electrophoresed $10 \%$ SDS-PAGE gels and then transferred onto PVDF membranes. Later on, membranes were incubated with primary antibodies specific to HIF-1 $\alpha$ (1:1000, Abcam), JAK2 (1:1000, Abcam), STAT3 (1:1000, Abcam), p-STAT3 (1:1000, Abcam), GAPDH (1:1000, Abcam) overnight at $4^{\circ} \mathrm{C}$, along with HRP-conjugated secondary antibody for 1 $\mathrm{h}$ at room temperature. Subsequently, the signals were visualized using an enhanced chemiluminescence kit (ASPEN).

\section{Exosome Labeling and Uptake}

Exosomes were fluorescently labeled using PKH26 membrane dye (Sigma). After that, labeled exosomes were cocultured with HUVECs for $24 \mathrm{~h}$ at $37^{\circ} \mathrm{C}$. Later on, exosomes that absorbed by HUVECs were observed by a fluorescence microscope. DAPI (blue) was used to label nuclei.

\section{Cell Counting Kit-8 (CCK-8) Assay}

HUVECs $\left(5 \times 10^{3}\right.$ cells) were plated onto 96-well plates overnight. After the treatment, the cells were treated with $10 \mu \mathrm{L}$ of CCK8 reagent (Dojindo Laboratories) for 20 min. Then, the absorbance values were analyzed by a SpectraMax microtiter plate reader (Molecular Devices) at $450 \mathrm{~nm}$.

\section{Wound Healing Assay}

HUVECs were seed onto 6-well plates at a density of $2 \times 10^{5}$ cells per well. After that, the wounds were made using $200-\mu \mathrm{L}$ pipette tips in the cell monolayer at $0 \mathrm{~h}$, and the cell monolayers were washed twice with PBS. Later on, DMEM media replaced with fresh serum-free media. Subsequently, the width of the wound area was captured at $0 \mathrm{~h}$ and $48 \mathrm{~h}$ by a microscope (Olympus).

\section{Transwell Migration Assay}

Transwell migration assay was performed using a 24-well transwell chamber (Corning). Briefly, the exosomestreated HUVECs suspended in serum-free DMEM (200 $\mu \mathrm{L})$ were seeded onto the upper chamber, and DMEM containing $10 \%$ FBS $(600 \mu \mathrm{L})$ was placed onto the lower chamber. After $24 \mathrm{~h}$ of incubation, cells on the lower surface were stained with $1 \%$ crystal violet. Then, the migrated cells were observed using a microscope.

\section{Tube Formation Assay}

Tube formation assay was performed using a 24-well transwell chamber pre-coated with Matrigel (Corning). Briefly, the exosomes-treated HUVECs $\left(1 \times 10^{5}\right.$ cells $)$ were plated onto the Matrigel-coated well for $24 \mathrm{~h}$. After that, tube formation was observed using a microscope, and the number of branches was measured using the IPP software.

\section{Luciferase Analysis}

The wild-type (wt) or mutant (mut) miR-216b-5p binding sites to SNHG1 or 3'-UTR of JAK2 were separately inserted into the pGL6-miR-based luciferase reporter vector (Beyotime), then co-transfected with miR-216b-5p mimics and NC mimic into cells. After $48 \mathrm{~h}$ of transfection, the luciferase activity was detected using the Dual Luciferase Reporter Assay System (Beyotime).

\section{Animal Study}

Female BALB/C nude mice were purchased from the Shanghai Laboratory Animal Center. Totally, 5×10 ${ }^{6}$ MDAMB-231 cells were subcutaneously injected into the left flank of nude mice. When the tumors reached about $200 \mathrm{~mm}^{3}$, animals were randomized into four groups: control, MDA-MB-231 Hypoxia Exo, MDA-MB-231 Hypoxia Exo $^{\text {siRNA-ctrl }}$ and MDA-MB-231 Hypoxia Exo ${ }^{\text {siSNHG1-3 }}$. Mice were intravenously injected with exosomes $(50 \mu \mathrm{g})$ twice a week. The nude mice were sacrificed on day 21 , the tumor tissues were removed completely. Then, tumor volume and weight were measured. All animal experiments were approved by the Ethics Committee of the Qilu Hospital of Shandong University, and animals were maintained following the institutional guidelines. In addition, the National Institutes of Health guide for the care and use of laboratory animals was followed.

\section{Statistical Analyses}

Data are expressed as the mean \pm standard deviation (SD). All statistical analyses were performed using GraphPad Prism software. Group comparisons were analyzed by oneway analysis of variance (ANOVA) and Tukey's tests. Differences between two groups were analyzed by twotailed Student's $t$-test. The differences were considered significant at $* \mathrm{P}<0.05$. All data were repeated in triplicate. 


\section{Results}

\section{SNHGI is Upregulated in Hypoxic Breast} Cancer Cell-Derived Exosomes

LncRNAs have been found to be participated in hypoxia signaling, thus contributing to tumor progression. ${ }^{25}$ As shown in Figure 1A, the expression of HIF-1 $\alpha$ was markedly increased in breast cancer cells under hypoxia $\left(\begin{array}{lll}1 \% & \mathrm{O}_{2}\end{array}\right)$, compared to cells cultured in normoxia. Moreover, the level of SNHG1 was significantly upregulated in breast cancer cells cultured under hypoxia comparing with that cultured under normoxia (Figure $1 \mathrm{~B}$ and $\mathrm{C}$ ). Next, exosomes were isolated from the medium of breast cancer cells cultured under normoxia or under hypoxia. As indicated in Figure 1D and $\mathrm{E}$, the isolated extracellular vesicles from MDA-MB-231 and MCF-7 cells were typical rounded, lipid bilayer membrane-encapsulated particles and had the characteristic size of exosomes (40 to $100 \mathrm{~nm}$ in diameter). It has been shown that hypoxia could promote tumor cells to release more exosomes. ${ }^{26}$ Consistently, the concentration of exosomes harvested from hypoxic MDA-MB-231 cells was significantly higher than that from normoxic MDA-MB-231 cells (Figure 1E). Moreover, the level of SNHG1 was increased in exosomes derived from hypoxic breast cancer cells compared with that derived normoxic breast cancer cells (Figure $1 \mathrm{~F}$ and $\mathrm{G}$ ). These data showed that SNHG1 is upregulated in exosomes derived from hypoxic breast cancer cells.

\section{SNHGI Can Be Transferred from Hypoxic Breast Cancer Cells to HUVECs via Exosomes}

It has been reported that exosomes derived from hypoxic cancer cells could promote the angiogenesis of HUVECs. $^{27}$ Thus, to investigate whether SNHG1 can be transferred from hypoxic breast cancer cells to HUVECs via exosomes, HUVECs were incubated with PKH26labeled exosomes that were derived from breast cancer cells under normoxic or hypoxic conditions. After 48 $\mathrm{h}$ of incubation, PKH26 fluorescence dye was observed in HUVECs. This outcome indicated the exosomes were absorbed by HUVECs (Figure 2A). In addition, the level of SNHG1 was notably increased in HUVECs incubated
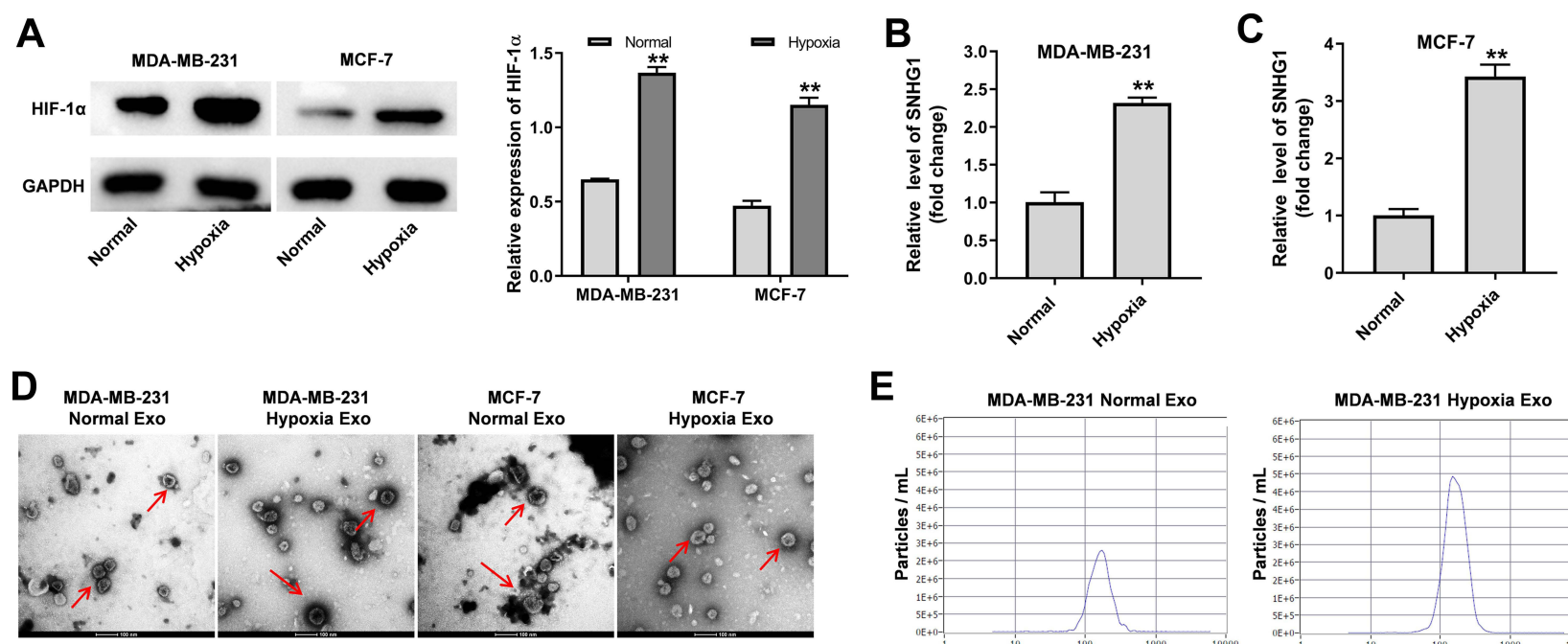

E
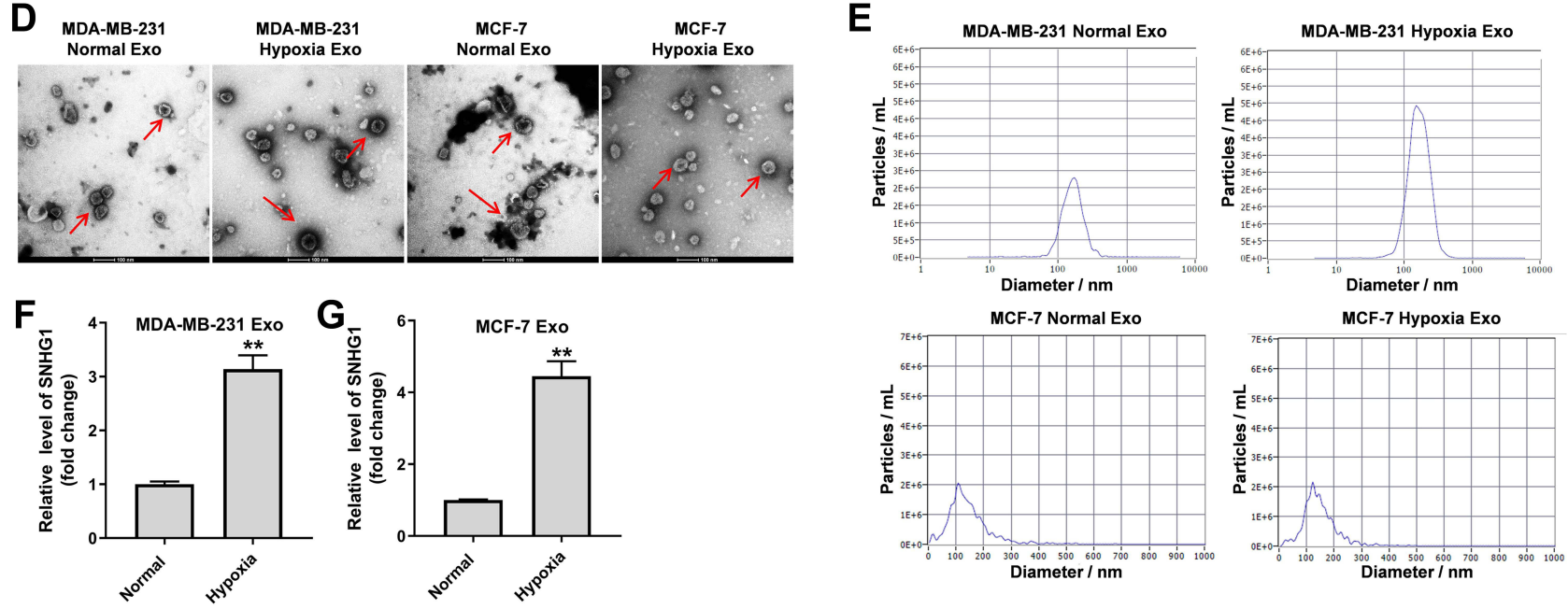

Figure I SNHGI is upregulated in exosomes derived from hypoxic breast cancer cells. MDA-MB-23I and MCF-7 cells were cultured for $24 \mathrm{~h}$ under hypoxia or normoxia. (A) Western blot analysis of HIF-I $\alpha$ level in MDA-MB-23I and MCF-7 cells. (B and C) RT-qPCR analysis of SNHGI level in MDA-MB-23I and MCF-7 cells. (D and E) Identification of exosomes derived from MDA-MB-23I and MCF-7 cells by TEM and NTA analysis. Exosome was indicated by the red arrow. (F and $\mathbf{G})$ RT-qPCR analysis of SNHGI level in exosomes derived from MDA-MB-23I and MCF-7 cells. Each experiment was repeated in triplicate $(n=3)$. **P<0.0I vs Normal group. 

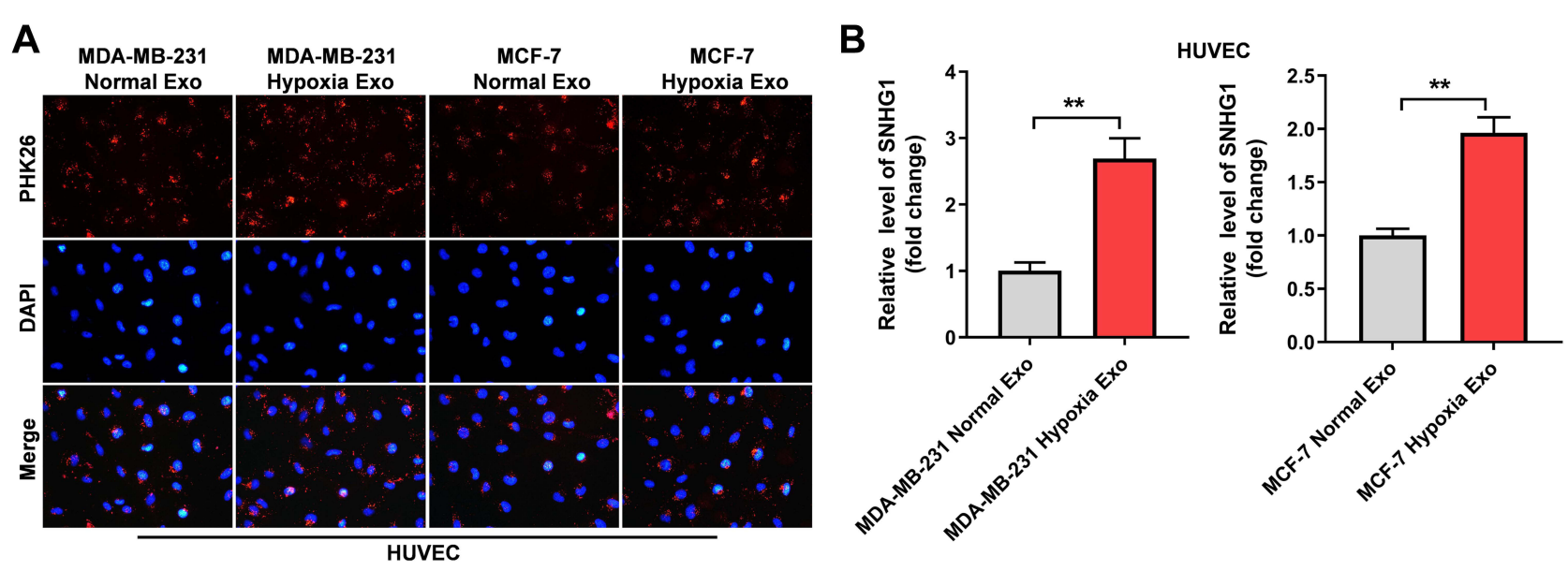

Figure 2 SNHGI can be transferred from hypoxic breast cancer cells to HUVECs via exosomes. (A) Exosomes were isolated from breast cancer cells under hypoxia or normoxia. Then, PKH26-labeled exosomes were co-cultured with HUVECs for $\mathbf{4 8} \mathrm{h}$, and the fluorescence signal was observed by microscopy. (B) Exosomes were isolated from breast cancer cells under hypoxia and normoxia. Then, RT-qPCR analysis of SNHGI level in HUVECs co-cultured with indicated exosomes. $* * P<0.0 \mathrm{I} ; \mathrm{n}=3$.

with exosomes derived from hypoxic breast cancer cells (Figure 2B). All these data suggested that SNHG1 could be transferred from hypoxic breast cancer cells to HUVECs via exosomes.

\section{Exosomes Derived from Hypoxic Breast Cancer Cells Promote the Proliferation, Migration and Angiogenesis of HUVECs}

To investigate the effects of exosomes derived from hypoxic breast cancer cells on endothelial cells,
CCK-8, wound healing, transwell assay and tube formation experiments were performed. As shown in Figure 3A-D, exosomes derived from hypoxic breast cancer cells remarkably promoted the proliferation, migration and angiogenesis of HUVECs compared with exosomes isolated from normoxic breast cancer cells (MDA-MB-231 or MCF7 normal Exo). Collectively, exosomes derived from hypoxic breast cancer cells were able to promote the proliferation, migration and angiogenesis of HUVECs.

A
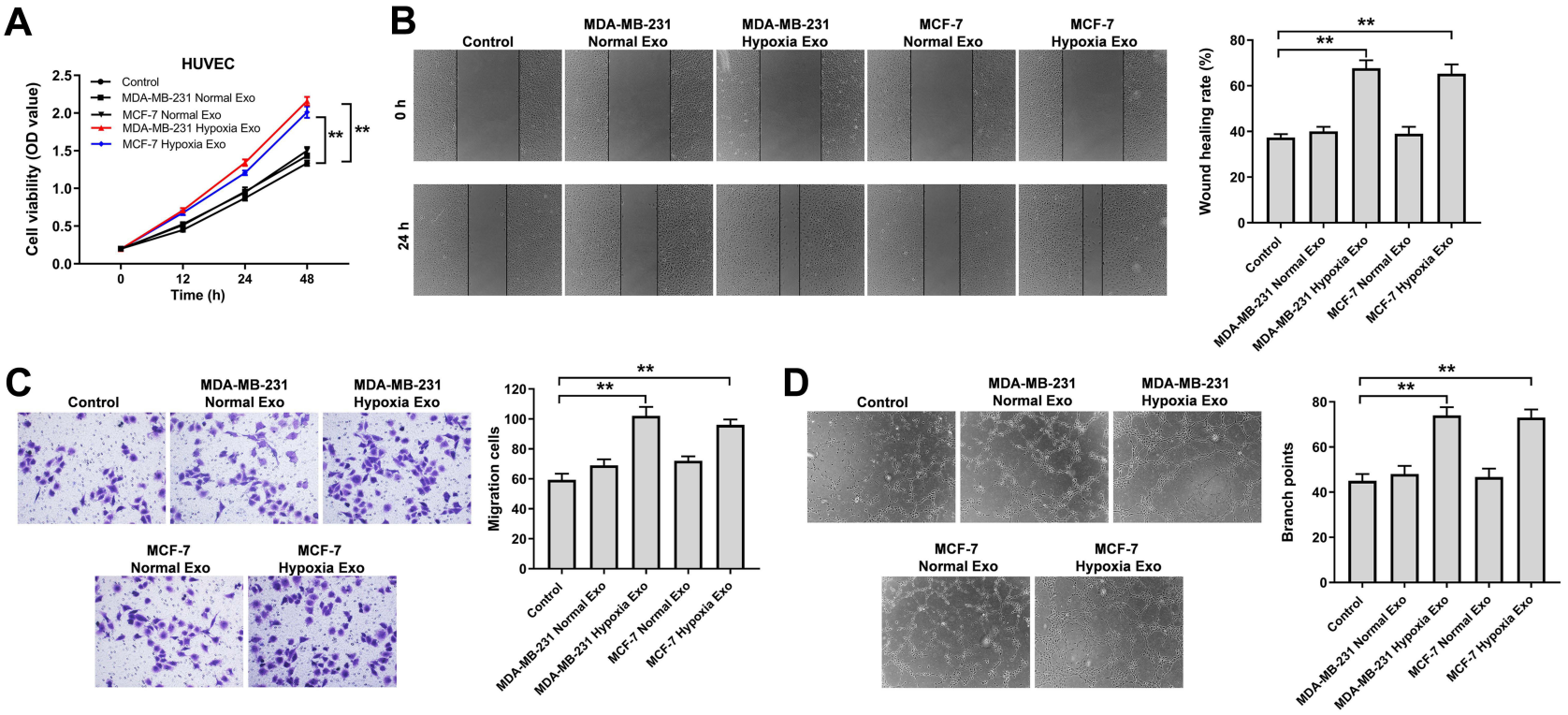

Figure 3 Exosomes derived from hypoxic breast cancer cells promoted the proliferation, migration and angiogenesis of HUVECs. Exosomes were isolated from breast cancer cells under hypoxia or normoxia. Then, HUVECs were co-cultured with indicated exosomes. (A) CCK-8 assay was applied to measure cell viability. (B) Wound healing assay was used to detect cell migration. (C) Transwell migration assay was used to detect cell migration. (D) Tube formation assay was applied to measure angiogenesis. $* * \mathrm{P}<0.01 ; \mathrm{n}=3$. 


\section{Exosomal SNHGI Derived from Hypoxic} Breast Cancer Cells Promotes the Migration and Angiogenesis of HUVECs

In order to explore the role of exosomal SNHG1 in tumorgenesis, three different siRNAs (SNHG1 siRNA1, SNHG1 siRNA2 and SNHG1 siRNA3) were used to downregulate the expression of SNHG1 in MDA-MB-231 cells (Figure 4A). Since SNHG1 siRNA3 exhibited the best inhibitory effect on SNHG1 expression compared with other siRNAs, SNHG1 siRNA3 was selected for use in the following experiments. In addition, exosomes derived from hypoxic MDA-MB-231 cells markedly increased the level of SNHG1 in HUVECs compared with MDA-MB -231 normal Exo; however, this change was notably reversed when hypoxic MDA-MB-231 cell-derived exosomes containing SNHG1 siRNA3 (Figure 4B). Moreover, hypoxic MDA-MB-231 cell-derived exosomes notably increased the migration and angiogenesis of HUVECs compared with MDA-MB-231 normal Exo; however, these phenomena were markedly inhibited when exosomes containing SNHG1 siRNA3 (Figure 4C-F). To sum up, exosomal SNHG1 derived from hypoxic breast cancer cells was able to promote the migration and angiogenesis of HUVECs.
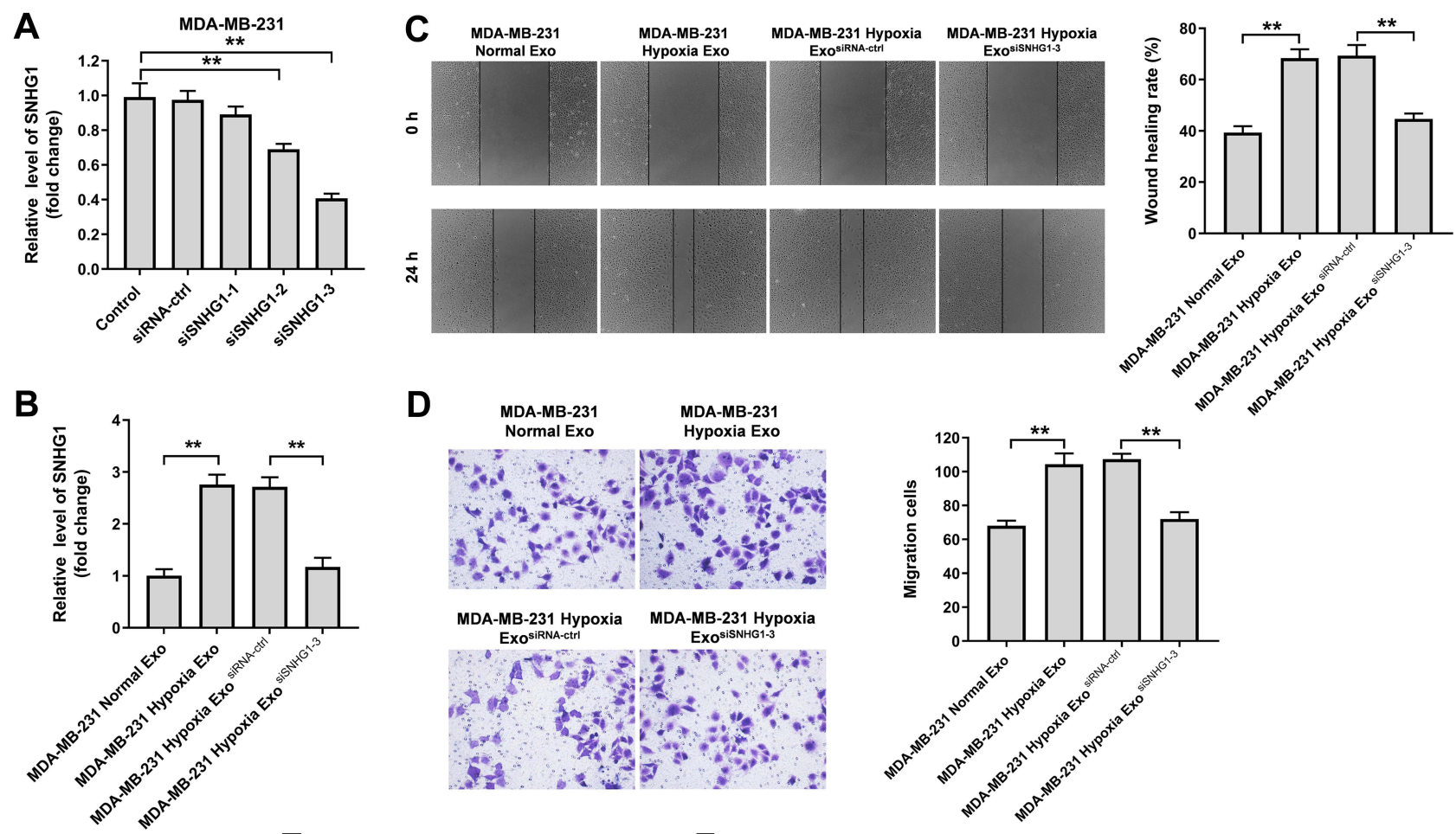

D
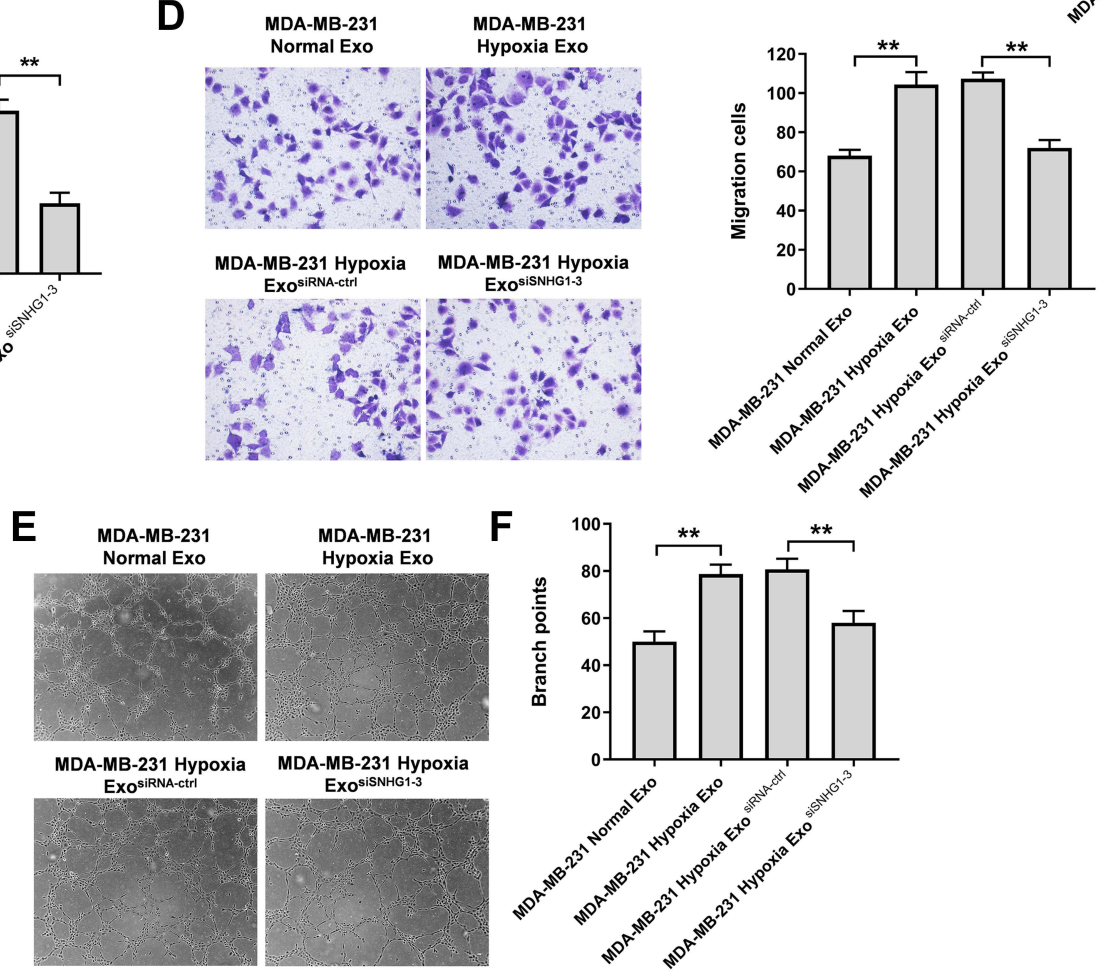

Figure 4 Exosomes derived from hypoxic breast cancer cells promotes the migration and angiogenesis of HUVECs. (A) RT-qPCR analysis of SNHGI level in MDA-MB-23I cells transfected with siRNA-ctrl, SNHGI siRNAI, siRNA2, siRNA3. (B) RT-qPCR analysis of SNHGI level in HUVECs co-cultured with exosomes isolated from hypoxic MDA-MB-23I cells. (C) Wound healing assay was used to detect cell migration. (D) Transwell migration assay was used to detect cell migration. (E and $\mathbf{F}$ ) Tube formation assay was applied to measure angiogenesis. $* * \mathrm{P}<0.01$. 


\section{Exosomes Derived from Hypoxic Breast Cancer Cells Promote the Migration of HUVECs via SNHGI/miR-2I6b-5p/JAK2 Axis}

To explore the mechanism by which hypoxic MDA-MB-231 cell-derived exosomes promoted the tumorgenesis, starBase (http://starbase.sysu.edu.cn) was used to predict the miRNAIncRNA interactions. As revealed in Figure 5A, a binding site was found between miR-216b-5p and SNHG1. In addition, previous studies reported the level of miR-216b-5p was downregulated in breast cancer cells and overexpression of miR-216-5p could suppress breast cancer growth, suggesting that miR-216b-5p is involved in the development of breast cancer. ${ }^{28,29}$ Thereby, we focus on investigating the relationship between SNHG1 and miR-216b-5p. As shown in Figure 5B, the luciferase activity of the SNHG1-WT plasmid was significantly decreased by miR-216b-5p mimics, suggesting that SNHG1 may be a direct binding target of miR-216b-5p. Moreover, the results of wound healing and transwell migration assay indicated that the migration promoting effects of hypoxic MDA-MB-231 cellderived exosomes on HUVECs were completely revered by miR-216b-5p mimics (Figure 5C and D).

Next, the data from TargetScan (http://www.targetscan. org/vert 71/) showed that JAK2 might be a potential binding target of miR-216b-5p (Figure 6A). In addition, evidence has shown that JAK2 is overexpressed in breast cancer cells. ${ }^{30}$ In addition, JAK2/STAT3 pathway plays an important role in breast cancer progression. ${ }^{31,32}$ Thus, dualluciferase reporter assay was used to explore the interaction between JAK2 and miR-216b-5p in the current study. As shown in Figure 6B, the luciferase activity of the JAK2-WT 3'UTR plasmid was markedly decreased by miR-216b-5p mimics, suggesting that JAK2 is a direct binding target of miR-216b-5p. Meanwhile, the expressions of JAK2 and p-STAT3 were upregulated in HUVECs incubated with exosomes derived from hypoxic MDA-MB-231 cells; however, these upregulations were notably reversed by miR-216b-5p mimics or by SNHG1 siRNA3 (Supplementary Figure 1A, $\underline{1 \mathrm{~B}}$ and Figure $6 \mathrm{C}-\mathrm{E})$. Collectively, exosomes derived from hypoxic breast cancer cells promote the migration of HUVECs via SNHG1/miR-216b-5p/JAK2 axis.

\section{Exosomes Derived from Hypoxic Breast Cancer Cells Promotes the Growth of Breast Cancer Cells in vivo}

Finally, we investigated the effect of hypoxic breast cancer cells-derived exosomes on the tumorigenicity of breast cancer in vivo. As shown in Figure 7A-C, hypoxic MDAMB-231 cell-derived exosomes markedly promoted the tumor volume and tumor weight of MDA-MB-231

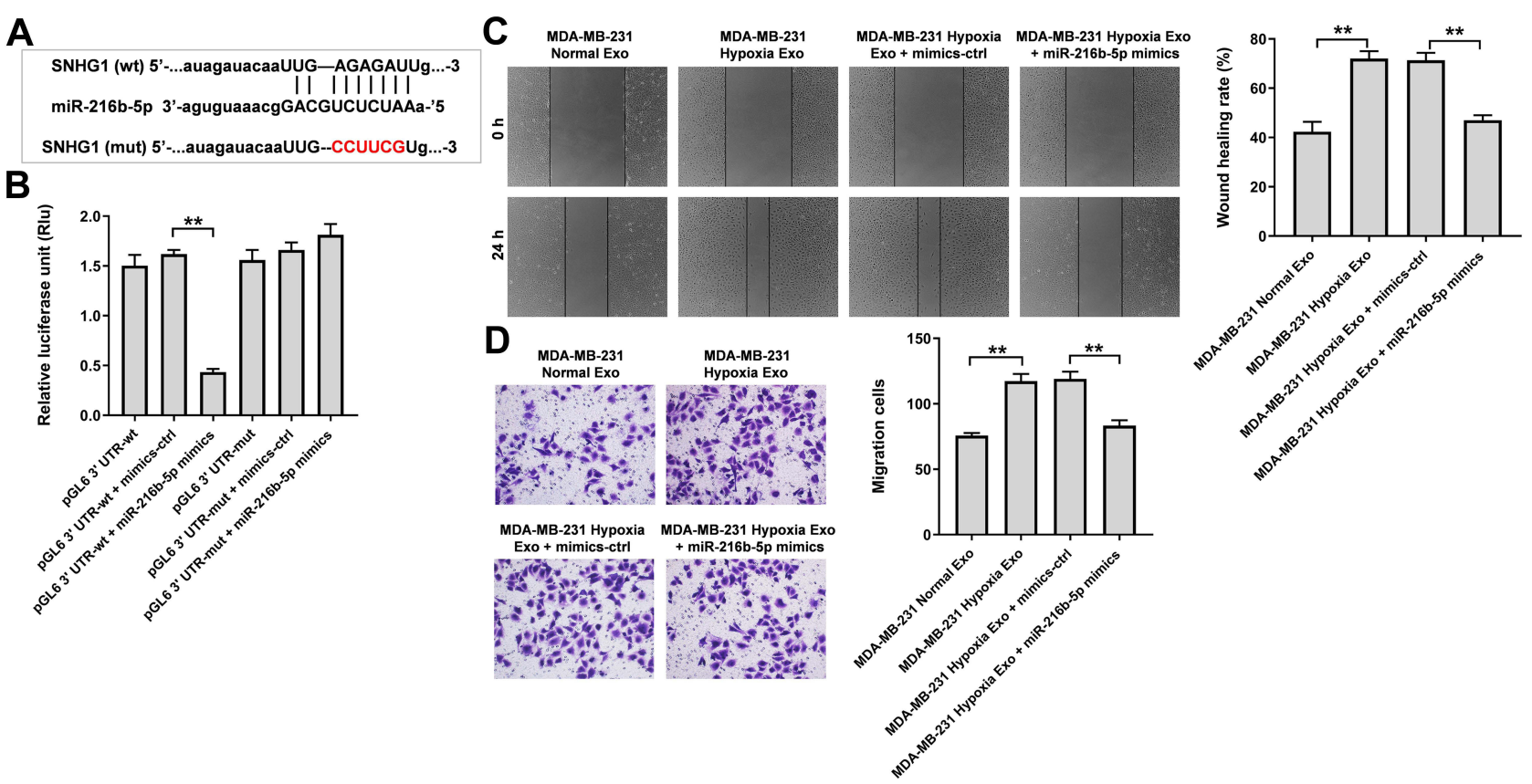

Figure 5 SNHGI acts as the sponge of miR-216b-5p. (A) Schematic diagram of binding sites between SNHGI and miR-2I6b-5p. (B) Luciferase activity analysis in 293T cells co-transfected with the SNHGI-wt/mut plasmids together with miR-2I6b-5p mimics. (C) HUVECs were treated with indicated exosomes, exosomes + mimics-ctrl or exosomes + miR-2I6b-5p mimics. Wound healing assay was used to detect cell migration. (D) Transwell migration assay was used to detect cell migration. $* * \mathrm{P}<0.0 \mathrm{I} ; \mathrm{n}=3$. 

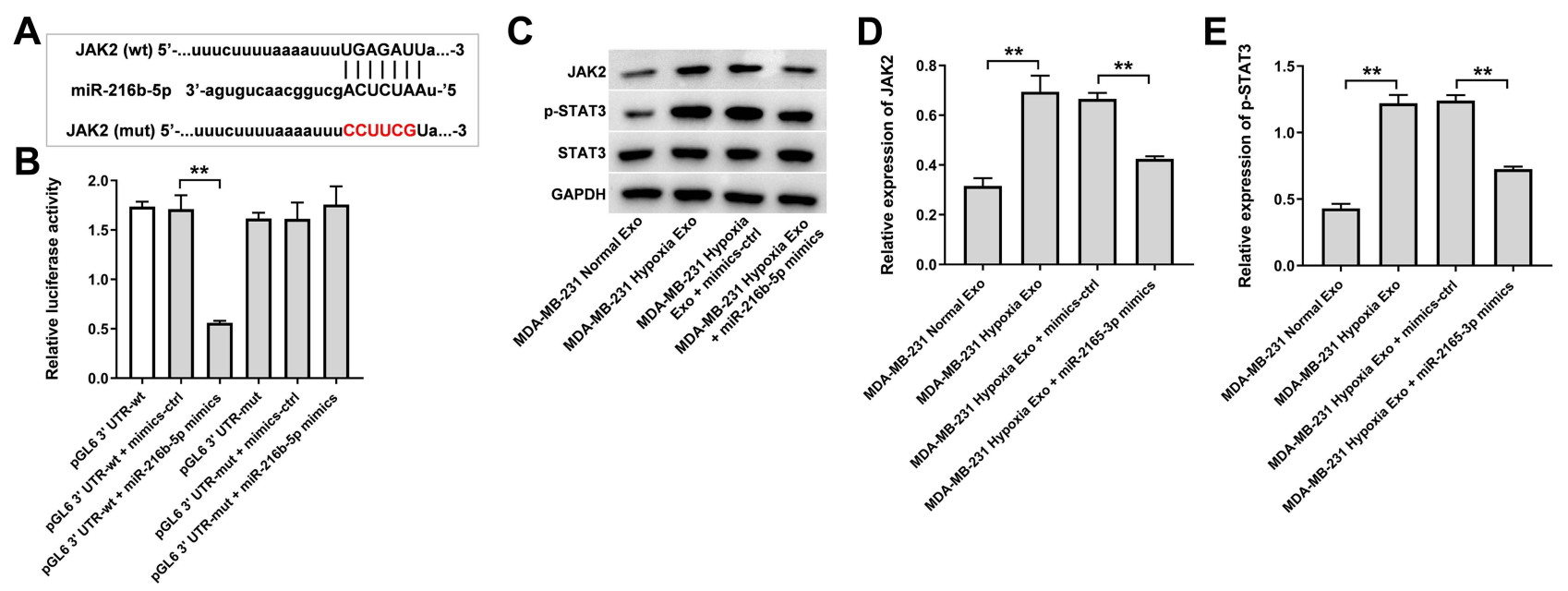

Figure 6 JAK2 is a direct binding target of miR-2I6b-5p. (A) Schematic diagram of binding sites between JAK2 and miR-2I6b-5p. (B) Luciferase activity analysis in 293T cells co-transfected with the JAK2-wt/mut plasmids together with miR-2I6b-5p mimics. (C-E) HUVECs were treated with indicated exosomes, exosomes + mimics-ctrl or exosomes + miR-216b-5p mimics. Western blot analysis of JAK2, p-STAT3 and STAT3 expressions in HUVECs. ${ }^{* * P}<0.01 ; n=3$.

subcutaneous xenografts compared with control group; however, these changes were reversed when exosomes containing SNHG1 siRNA3. In addition, hypoxic MDAMB-231 cell-derived exosomes significantly increased SNHG1 level and JAK2 and p-STAT3 protein expressions in tumor tissues; however, these increases were all reversed when exosomes containing SNHG1 siRNA3 (Figure 7D-G). Collectively, exosomal SNHG1 derived from hypoxic breast cancer cells significantly promotes the growth of breast cancer cells in vivo.
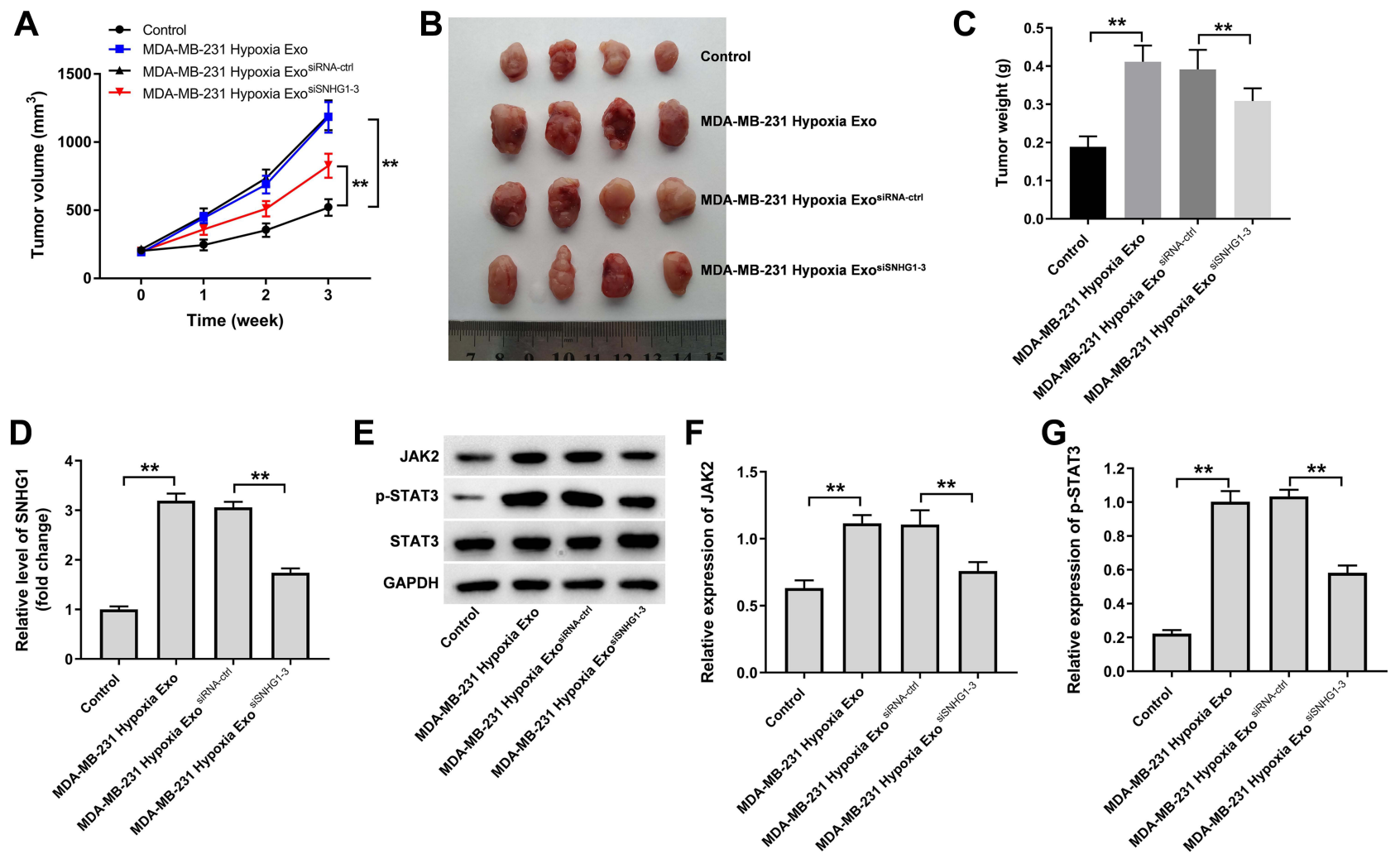

Figure 7 Exosomal SNHGI derived from hypoxic breast cancer cells promotes the growth of breast cancer cells in vivo. (A-C) Tumor volume and tumor weight of xenograft tumors. (D) RT-qPCR analysis of SNHGI level in tumor tissues. (E-G) Western blot analysis of JAK2, p-STAT3 and STAT3 expressions in tumor tissues. **P $<0.0 \mathrm{I}$; $\mathrm{n}=3$. 


\section{Discussion}

Exosomes containing informative miRNAs have been shown to be participated in cell-to-cell communication between cancer cells and recipient cells, thereby contributing to remodeling of the TME. ${ }^{27}$ In addition, hypoxia could promote the release of exosomes from tumor cells. $^{26,33}$ In this study, we found that SNHG1 and HIF- $\alpha$ were upregulated in breast cancer cells under hypoxia. Importantly, SNHG1 can be transferred from hypoxic breast cancer cells to endothelial cells via exosomes, then induces the cell migration and angiogenesis, and consequently contributes to breast cancer progression.

Accumulating evidences have shown that SNHG1 plays important roles in the carcinogenesis and progression of various cancers. ${ }^{20,34}$ SNHG1 is upregulated and acts as an oncogene in cervical cancer. ${ }^{34}$ In addition, SNHG1 promoted cell migration and invasion of bladder cancer by functioning as a carcinogenic lncRNA. ${ }^{35}$ Meanwhile, SNHG1 was able to promote breast cancer progression via regulating miR-573/LMO4 axis. ${ }^{23}$ More importantly, Zuo et al found that SNHG1 level was increased under hypoxic condition in breast cancer in a HIF-1-dependent manner ${ }^{24}$; hypoxia-induced lncRNAs could induce tumor invasion, metastasis and angiogenesis. $^{36,37}$ In line with the findings in the above studies, we found that SNHG1 level was increased in breast cancer cells under hypoxia condition. Additionally, SNHG1 was highly expressed in exosomes derived from hypoxic breast cancer cells and they could be transferred from breast cancer cells to HUVECs via exosomes. Meanwhile, hypoxic breast cancer cells-derived exosome notably promoted the proliferation, migration and angiogenesis of HUVECs, whereas these effects were abolished when hypoxic breast cancer cell-derived exosomes containing SNHG1 siRNA. Guo et al found that hypoxic pancreatic cancer cells-derived exosomal UCA1 promoted tumor angiogenesis by targeting miR-96-5p/AMOTL2 axis. ${ }^{19}$ All these data suggested that exosomes-mediated transfer of lncRNA between hypoxic breast cancer cells and endothelial cells is important for breast cancer progression.

It has been shown that IncRNA could serve as sponges of miRNAs, decreased their regulatory effect on mRNAs. ${ }^{38}$ To explore whether SNHG1 could regulate the progression of breast cancer via sponging miRNAs, starBase database was used in the current study. We found that miR-216b-5p might be sponged by SNHG1, and the interaction between miR-216b-5p and SNHG1 was confirmed by luciferase activity assay. Previous studies showed that miR-216b markedly inhibited colorectal cancer cell proliferation, migration and angiogenesis. ${ }^{39}$ In addition, overexpression of miR-216b significantly suppressed cervical cancer cell proliferation by targeting FOXM1. ${ }^{40}$ These data indicated the antitumor roles of miR-216b by inhibiting the proliferation, migration and angiogenesis in human cancers. Rescue experiments revealed that overexpression of miR-216b-5p attenuated the pro-angiogenesis effect of hypoxic breast cancer cells-derived exosomes in HUVECs, indicating that exosomal SNHG1 might regulate the angiogenesis of breast cancer by sponging miR-216b-5p.

JAK/STAT pathway plays an essential role in the tumorigenesis, metastasis and angiogenesis of breast cancer. ${ }^{41,42}$ In addition, hypoxia could contribute to the tumor progression via activation of JAK/STAT pathway. ${ }^{43}$ In this study, we found that JAK2 was a target of miR-216b-5p, which was confirmed by luciferase activity assay as well. Hypoxic breast cancer cell-derived exosomes significantly upregulated the expressions of JAK2 and p-STAT3 in HUVECs; however, these changes were reversed by miR-216b-5p mimics. Moreover, hypoxic breast cancer cells-derived exosomes markedly promoted tumor growth in vivo via upregulation of JAK2/STAT3 pathway. These results suggested that exosomal SNHG1 might regulate the breast cancer growth and angiogenesis of by targeting miR-216b-5p/JAK2 axis.

\section{Conclusion}

In this study, we found that exosomal SNHG1 from hypoxic breast cancer cells could promote tumor growth and angiogenesis via regulating miR-216b-5p/JAK2 axis, suggesting that SNHG1 may serve as a potential therapeutic target for the treatment of breast cancer.

\section{Data Sharing Statement}

The datasets used and/or analyzed during the current study are available from the corresponding author on reasonable request.

\section{Ethics Approval and Consent to Participate}

All animal experiments were approved by the Ethics Committee of the Qilu Hospital of Shandong University, and animals were maintained following the institutional guidelines. 


\section{Funding}

There is no funding to report.

\section{Disclosure}

The authors declare no conflict of interest.

\section{References}

1. Fahad Ullah M. Breast cancer: current perspectives on the disease status. Adv Exp Med Biol. 2019;1152:51-64.

2. Yin Z, Wang W, Qu G, et al. MiRNA-96-5p impacts the progression of breast cancer through targeting FOXO3. Thorac Cancer. 2020;11 (4):956-963. doi:10.1111/1759-7714.13348

3. Xue J, Chi Y, Chen Y, et al. MiRNA-621 sensitizes breast cancer to chemotherapy by suppressing FBXO11 and enhancing p53 activity. Oncogene. 2016;35(4):448-458. doi:10.1038/onc.2015.96

4. Ding L, Gu H, Xiong X, et al. MicroRNAs involved in carcinogenesis, prognosis, therapeutic resistance and applications in human triple-negative breast cancer. Cells. 2019;8(12):1492. doi:10.3390/ cells 8121492

5. Krug D, Baumann R, Budach W, et al. Current controversies in radiotherapy for breast cancer. Radiat Oncol. 2017;12(1):25

6. He R, Liu P, Xie X, et al. circGFRA1 and GFRA1 act as ceRNAs in triple negative breast cancer by regulating miR-34a. $J$ Exp Clin Cancer Res. 2017;36(1):145. doi:10.1186/s13046-017-0614-1

7. Erić I, Petek erić A, Koprivčić I, et al. Independent factors FOR poor prognosis in young patients with stage I-III breast cancer. Acta Clin Croat. 2020;59(2):242-251. doi:10.20471/acc.2020.59.02.07

8. Yin Z, Li C, Wang J, Xue L. Myeloid-derived suppressor cells: roles in the tumor microenvironment and tumor radiotherapy. Int $J$ Cancer. 2019;144(5):933-946. doi:10.1002/ijc.31744

9. Hui L, Chen Y. Tumor microenvironment: sanctuary of the devil. Cancer Lett. 2015;368(1):7-13. doi:10.1016/j.canlet.2015.07.039

10. Jing X, Yang F, Shao C, et al. Role of hypoxia in cancer therapy by regulating the tumor microenvironment. Mol Cancer. 2019;18 (1):157. doi:10.1186/s12943-019-1089-9

11. Rana NK, Singh P, Koch B. CoCl(2) simulated hypoxia induce cell proliferation and alter the expression pattern of hypoxia associated genes involved in angiogenesis and apoptosis. Biol Res. 2019;52 (1):12. doi:10.1186/s40659-019-0221-z

12. Zhang C, Samanta D, Lu H, et al. Hypoxia induces the breast cancer stem cell phenotype by HIF-dependent and ALKBH5-mediated $\mathrm{m}^{6}$ A-demethylation of NANOG mRNA. Proc Natl Acad Sci U S A. 2016;113(14):E2047-2056. doi:10.1073/pnas.1602883113

13. Yu DD, Wu Y, Shen HY, et al. Exosomes in development, metastasis and drug resistance of breast cancer. Cancer Sci. 2015;106 (8):959-964. doi:10.1111/cas.12715

14. Kalluri R. The biology and function of exosomes in cancer. $J$ Clin Invest. 2016;126(4):1208-1215. doi:10.1172/JCI81135

15. Shao C, Yang F, Miao S, et al. Role of hypoxia-induced exosomes in tumor biology. Mol Cancer. 2018;17(1):120. doi:10.1186/s12943018-0869-y

16. Zeltz C, Primac I, Erusappan P, et al. Cancer-associated fibroblasts in desmoplastic tumors: emerging role of integrins. Semin Cancer Biol. 2020;62:166-181. doi:10.1016/j.semcancer.2019.08.004

17. Mao Y, Wang Y, Dong L, et al. Hypoxic exosomes facilitate angiogenesis and metastasis in esophageal squamous cell carcinoma through altering the phenotype and transcriptome of endothelial cells. J Exp Clin Cancer Res. 2019;38(1):389. doi:10.1186/s13046019-1384-8

18. Xue M, Chen W, Xiang A, et al. Hypoxic exosomes facilitate bladder tumor growth and development through transferring long non-coding RNA-UCA1. Mol Cancer. 2017;16(1):143. doi:10.1186/s12943-0170714-8
19. Guo Z, Wang X, Yang Y, et al. Hypoxic tumor-derived exosomal long noncoding RNA UCA1 promotes angiogenesis via miR-96-5p/ AMOTL2 in pancreatic cancer. Mol Ther Nucleic Acids. 2020;22:179-195. doi:10.1016/j.omtn.2020.08.021

20. Xu M, Chen X, Lin K, et al. The long noncoding RNA SNHG1 regulates colorectal cancer cell growth through interactions with EZH2 and miR-154-5p. Mol Cancer. 2018;17(1):141. doi:10.1186/ s12943-018-0894-x

21. Zheng S, Li M, Miao K, Xu H. SNHG1 contributes to proliferation and invasion by regulating miR-382 in breast cancer. Cancer Manag Res. 2019;11:5589-5598. doi:10.2147/CMAR.S198624

22. Meng XF, Liu AD, Li SL. SNHG1 promotes proliferation, invasion and EMT of prostate cancer cells through miR-195-5p. Eur Rev Med Pharmacol Sci. 2020;24(19):9880-9888. doi:10.26355/ eurrev_202010_23198

23. Xiong $\mathrm{X}$, Feng Y, Li L, et al. Long non-coding RNA SNHG1 promotes breast cancer progression by regulation of LMO4. Oncol Rep. 2020;43(5):1503-1515. doi:10.3892/or.2020.7530

24. Zuo Y, Qu C, Tian Y, et al. The HIF-1/SNHG1/miR-199a-3p/TFAM axis explains tumor angiogenesis and metastasis under hypoxic conditions in breast cancer. Biofactors. 2021. doi:10.1002/biof.1702

25. Huan L, Guo T, Wu Y, et al. Hypoxia induced LUCAT1/PTBP1 axis modulates cancer cell viability and chemotherapy response. Mol Cancer. 2020;19(1):11. doi:10.1186/s12943-019-1122-z

26. Lu Q, Wang X, Zhu J, et al. Hypoxic tumor-derived exosomal Circ0048117 facilitates M2 macrophage polarization acting as miR-140 sponge in esophageal squamous cell carcinoma. Onco Targets Ther. 2020;13:11883-11897. doi:10.2147/OTT.S284192

27. Wu F, Li F, Lin $\mathrm{X}$, et al. Exosomes increased angiogenesis in papillary thyroid cancer microenvironment. Endocr Relat Cancer. 2019;26(5):525-538. doi:10.1530/ERC-19-0008

28. Menbari MN, Rahimi K, Ahmadi A, et al. MiR-216b-5p inhibits cell proliferation in human breast cancer by down-regulating HDAC8 expression. Life Sci. 2019;237:116945. doi:10.1016/j.lfs.2019.116945

29. Jana S, Sengupta S, Biswas S, et al. miR-216b suppresses breast cancer growth and metastasis by targeting SDCBP. Biochem Biophys Res Commun. 2017;482(1):126-133.

30. Wang X, Qiu W, Zhang G, et al. MicroRNA-204 targets JAK2 in breast cancer and induces cell apoptosis through the STAT3/BCl-2/ survivin pathway. Int J Clin Exp Pathol. 2015;8(5):5017-5025.

31. Doheny D, Sirkisoon S, Carpenter RL, et al. Combined inhibition of JAK2-STAT3 and SMO-GLI1/tGLI1 pathways suppresses breast cancer stem cells, tumor growth, and metastasis. Oncogene. 2020;39 (42):6589-6605. doi:10.1038/s41388-020-01454-1

32. Lin WH, Dai WG, Xu XD, et al. Downregulation of DPF3 promotes the proliferation and motility of breast cancer cells through activating JAK2/STAT3 signaling. Biochem Biophys Res Commun. 2019;514 (3):639-644. doi:10.1016/j.bbrc.2019.04.170

33. Kumar A, Deep G. Hypoxia in tumor microenvironment regulates exosome biogenesis: molecular mechanisms and translational opportunities. Cancer Lett. 2020;479:23-30. doi:10.1016/j. canlet.2020.03.017

34. Liu Y, Yang Y, Li L, et al. LncRNA SNHG1 enhances cell proliferation, migration, and invasion in cervical cancer. Biochem Cell Biol. 2018;96(1):38-43. doi:10.1139/bcb-2017-0188

35. Du Q, Chen J. SNHG1 promotes proliferation, migration and invasion of bladder cancer cells via the PI3K/AKT signaling pathway. Exp Ther Med. 2020;20(5):110. doi:10.3892/etm.2020.9238

36. Zhang J, Jin HY, Wu Y, et al. Hypoxia-induced LncRNA PCGEM1 promotes invasion and metastasis of gastric cancer through regulating SNAI1. Clin Transl Oncol. 2019;21(9):1142-1151. doi:10.1007/ s12094-019-02035-9

37. Niu Y, Bao L, Chen Y, et al. HIF2-induced long noncoding RNA RAB11B-AS1 promotes hypoxia-mediated angiogenesis and breast cancer metastasis. Cancer Res. 2020;80(5):964-975. doi:10.1158/ 0008-5472.CAN-19-1532 
38. Paraskevopoulou MD, Hatzigeorgiou AG. Analyzing MiRNA-LncRNA interactions. Methods Mol Biol. 2016;1402:271-286.

39. Chen X, Liu X, He B, et al. MiR-216b functions as a tumor suppressor by targeting HMGB1-mediated JAK2/STAT3 signaling way in colorectal cancer. Am J Cancer Res. 2017;7 (10):2051-2069.

40. He S, Liao B, Deng Y, et al. MiR-216b inhibits cell proliferation by targeting FOXM1 in cervical cancer cells and is associated with better prognosis. BMC Cancer. 2017;17(1):673. doi:10.1186/ s12885-017-3650-5
41. Shao F, Pang X, Baeg GH. Targeting the JAK/STAT signaling pathway for breast cancer. Curr Med Chem. 2020;28:5137-5151.

42. Zeng P, Sun S, Li R, Xiao ZX, Chen H. HER2 upregulates ATF4 to promote cell migration via activation of ZEB1 and downregulation of E-Cadherin. Int $J$ Mol Sci. 2019;20(9):2223. doi:10.3390/ ijms 20092223

43. Lee MY, Joung YH, Lim EJ, et al. Phosphorylation and activation of STAT proteins by hypoxia in breast cancer cells. Breast. 2006;15 (2):187-195. doi:10.1016/j.breast.2005.05.005

\section{Publish your work in this journal}

Cancer Management and Research is an international, peer-reviewed open access journal focusing on cancer research and the optimal use of preventative and integrated treatment interventions to achieve improved outcomes, enhanced survival and quality of life for the cancer patient.
The manuscript management system is completely online and includes a very quick and fair peer-review system, which is all easy to use. Visit http://www.dovepress.com/testimonials.php to read real quotes from published authors. 